\title{
Multi-objective Optimization of Variable-stiffness Composites Fabricated by Tailored Fiber Placement Machine
}

\author{
Shinya Honda ${ }^{\mathrm{a}, *}$

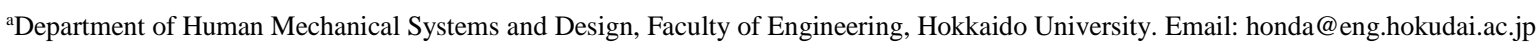

\begin{abstract}
A multi-objective optimization method for the laminated composite fabricated by a tailored fiber placement machine that is an application of embroidering machine is presented. The mechanical properties of composite with curvilinear fibers including stiffness, volume fraction, and density are variable depending on curvatures of fibers. The present study first measures the relation between curvatures and mechanical properties. The measured results indicate that the stiffness of composite decreases linearly as the curvature increases. Then, the obtained relation is applied to the multi-objective optimization where the maximum principal strain and magnitude of curvature are employed as objective functions. Obtained Pareto optimum solutions are widely distributed ranging from the solutions with curvilinear fibers to those with straight fibers, and the curvilinear fiber has still advantages over straight fiber even its weakened stiffness.
\end{abstract}

Keywords: Laminated composite; multi-objective optimization; variable-stiffness composites; tailored fiber placement

\section{Introduction}

Due to the development of fabrication process of composite materials, it has become possible to produce composites with curved reinforcing fibers. Since curvilinear fibers indicate locally varying anisotropic property in the same layer, it is possible to design with larger degree-of-freedom in terms of anisotropy compared with straight fibers. This results in that the laminated composites with optimally designed curved reinforcing fibers possess higher mechanical properties to the specific occasion than the conventional composite with straight fibers.

Many studies about composites with curvilinear fibers are reported in the past [1-5], and the authors also propose an optimization method of composite with curvilinear fibers $[6,7]$ where it was revealed that there is a trade-off relationship between mechanical properties and curvature of reinforcing fibers. Moreover, it was also found from experimental studies by using a tailored fiber placement (TFP) machine that the fiber volume fraction and layer thickness varies depending on curvature since fiber tows shrink and become thick along the curved path. This causes a gap between fiber tows and overlapping of adjacent tows.

The present study first reveals the relationship among curvature, volume fraction, and stiffnesses of composite with curved fibers by measuring densities of test

${ }^{*}$ Corresponding author. Tel.: +81-11-706-6415

Kita-13, Nishi-8, Kita-ku

Sapporo, Hokkaido, Japan, 060-8628 specimens with various curvatures fabricated by the TFP machine. Then, obtained relationship is applied to the multi-objective optimization problem that has two conflicting objective functions including in-plane mechanical properties and fiber curvatures.

\section{Experiment}

\subsection{Experimental methods}

The tailored fiber placement (TFP) machine is employed to fabricate composites with curvilinear fibers. The TFP machine is an application of embroidering machine and its sewing head is shown in Fig. 1 (a). Fiber tows (TR50S12L) are provided from horizontally rotating bobbin, and they are fixed by sewing thread in a zigzag manner to the base material as indicated in Fig. 1 (b) where peel-ply cross is used as a base material. Then, the fabricated preform is molded by using the vacuumed resign transfer molding (VaRTM) method with unsaturated polyester.

Six kinds of test specimens with constant curvatures are prepared to reveal the relationship between curvature and fiber volume fraction. The dimension of composite is $150 \mathrm{~mm} \times 150 \mathrm{~mm}$ with four layers, and fiber shapes are defined by an equation of arc, $y=-\sqrt{R^{2}-x^{2}}$. Distance between adjacent tow is $2.5 \mathrm{~mm}$ at the center line of plate. Curvatures of each specimen are $\kappa=1 / 100,1 / 150,1 / 200$, $1 / 250,1 / 300$, and $0 \mathrm{~mm}^{-1}$ (straight). As an example, design of fiber shape and preform before molding are indicated in Fig. 2(a) and (b). 


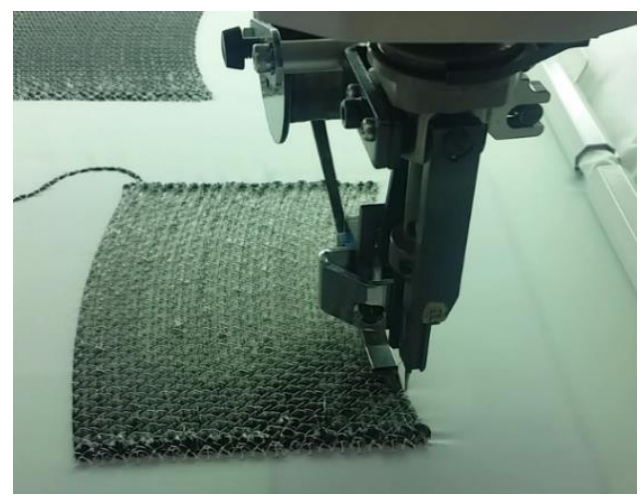

(a) Sewing head

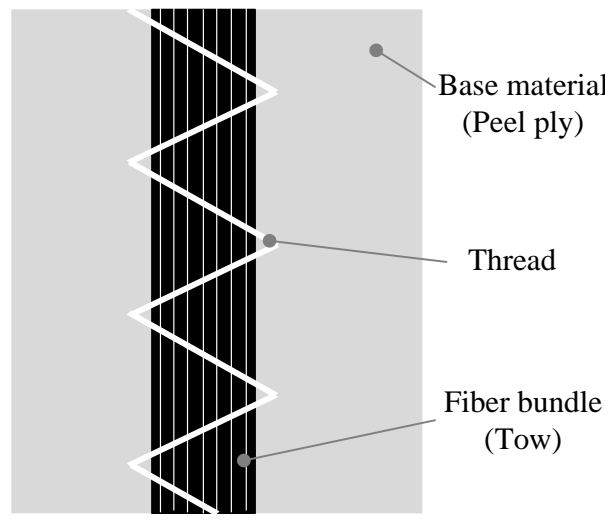

(b) Fiber tow and zigzag thread

Figure 1. Sewing head of TFP machine and schematic diagram of fiber placement

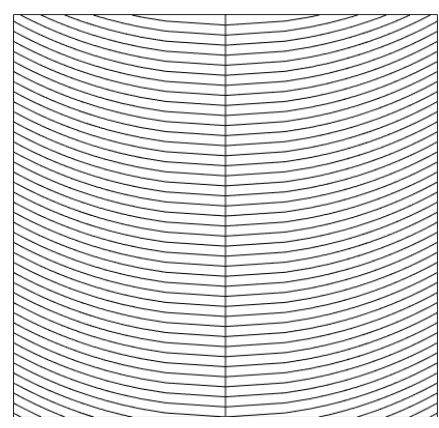

(a) Fiber design

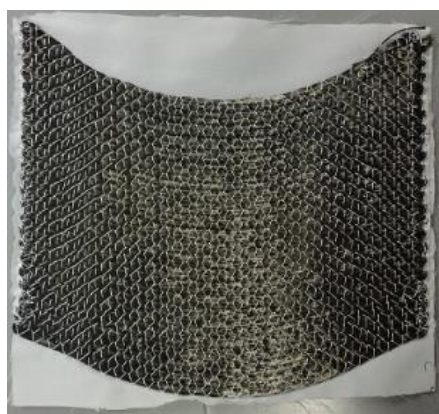

(b) Preform

Figure 2. Example of (a) fiber shape and (b) preform of test specimen $\kappa$ $=1 / 100 \mathrm{~mm}^{-1}$

\subsection{Experimental results}

Figure 3 indicates measured relation between (a) thickness and curvature, and (b) density and curvature. Three test specimens are prepared for each curvature. Blue diamonds show averaged values and error bars are standard deviations. Red lines are linear regression equations for both results and they are determined as follows.

$$
\begin{gathered}
h=30.0 \kappa+2.10[\mathrm{~mm}] \\
\rho_{c}=-5.9 \times 10^{3} \kappa+1405\left[\mathrm{~kg} / \mathrm{m}^{3}\right]
\end{gathered}
$$

where $\rho_{c}$ is a density of composite. Coefficients of determination are 0.814 and 0.917 , respectively. Using Eq. (2) and the mixture rule of composites, the fiber volume fraction $V_{f}$ is estimated with densities of fiber $\rho_{f}=$ $1820 \mathrm{~kg} / \mathrm{m}^{3}$ and matrix $\rho_{m}=1180 \mathrm{~kg} / \mathrm{m}^{3}$. Finally, variable stiffnesses $E_{1}$ and $E_{2}$ and Poisson's ratio $v_{12}$ depending on curvature are calculated by using mixture rule again. As with the density, the stiffness decreases linearly as the curvature increases. That is, a part with curved fiber has lower mechanical properties than straight ones. In the next chapter, it will be confirmed by the optimization problem if the curvilinear fiber nevertheless has advantages over straight fibers.

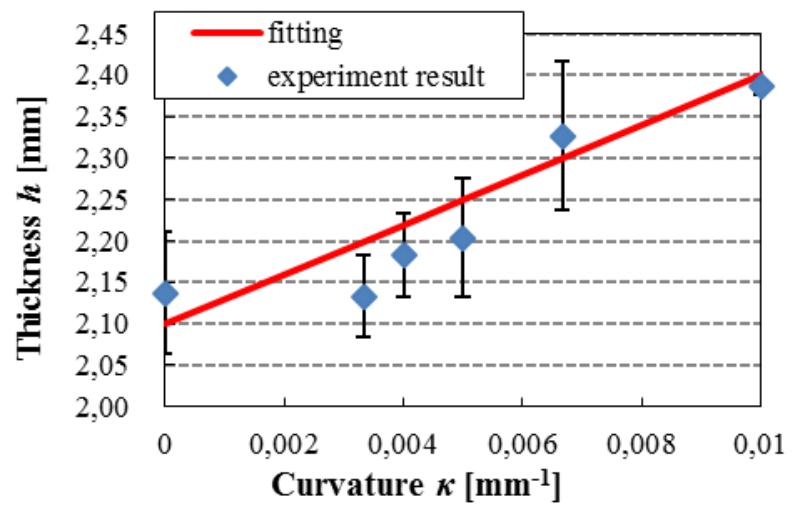

(a) Thickness vs curvature

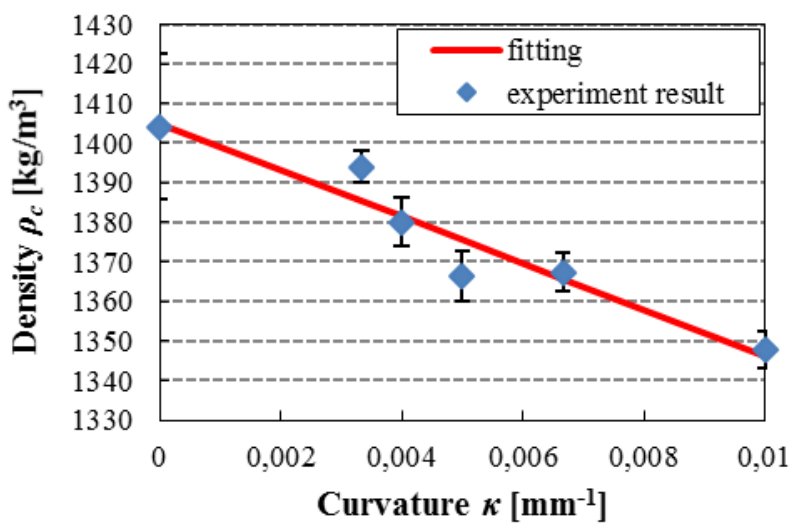

(b) Density vs curvature

Figure 3. Experimentally obtained relationship between (a) thickness and curvature, and (b) density and curvature 


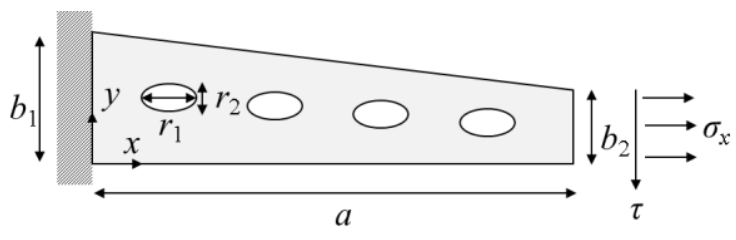

(a) Dimensions and loading condition

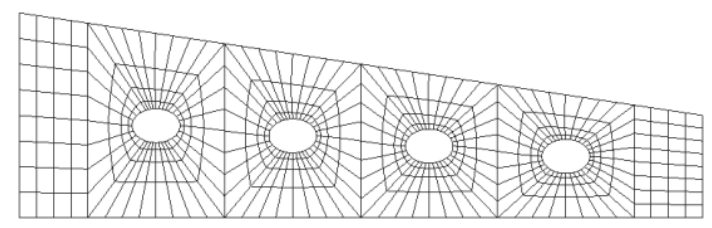

(b) Meshing

Figure 4. Numerical model for the optimization

\section{Optimization}

\subsection{Optimization conditions}

The present study employs a cantilever wing-shaped plate with four elliptical holes under in-plane loading as an optimized numerical model (Fig. 4 (a)). Its dimensions are $a=1000 \mathrm{~mm}, b_{1}=300 \mathrm{~mm}, b_{2}=150 \mathrm{~mm}, r_{1}=$ $70 \mathrm{~mm}, r_{2}=50 \mathrm{~mm}$, and each elliptical hole is located at $x=200,400,600,800 \mathrm{~mm}$, respectively. Loading conditions are $\sigma_{x}=175 \mathrm{MPa}, \tau=17.5 \mathrm{MPa}$. The number of layer is fixed to 16 . The finite element model is shown in Fig. 4(b), and numbers of element and node result in 576 and 1949.

For an expression of arbitral curvilinear reinforcing fibers, a surface defined by a cubic polynomial function is employed as follows [6-7].

$$
\begin{aligned}
f(x, y)=c_{10} x & +c_{01} y+c_{20} x^{2}+c_{11} x y \\
& +c_{02} y^{2}+c_{30} x^{3}+c_{21} x^{2} y \\
& +c_{12} x y^{2}+c_{03} y^{3}
\end{aligned}
$$

For example, Eq. (3) gives a surface shown in Fig. 5(a). Contour lines of the surface projected to the horizontal plane is indicated in Fig. 5 (b). By varying the values of $c_{i j}(i, j=1,2,3)$ arbitrary, various shapes of curves are expressed with Eq. (3), and these coefficients are employed as the design variables in the optimization. When these curved fibers are implemented in the finite element analysis, tangential directions of contour lines are calculated at center of each element by following equation.

$$
\theta_{n, p}\left(x_{c}, y_{c}\right)=\left.\tan ^{-1}\left(-\frac{\partial f / \partial x}{\partial f / \partial y}\right)\right|_{x=x_{c}, y=y_{c}}
$$

where $x_{c}$ and $y_{c}$ is the coordinate of center of element, and $\theta_{n, p}$ is the fiber orientation angle at $p$-th element in the $n$-th layer. Thus, curved fibers are discretized and approximated by the continuously changing short straight fibers in the FEA. Figure 6 (a) shows discrete fiber shape corresponding with Fig. 5. This fiber shape defined by Eq. (3) explicitly is referred as $+\theta$ layer, and a fiber shape which has symmetric fiber shape with respect to horizontal line at each element is $-\theta$ layer as shown in Fig. 6 (b).

Then, the present optimization problem can be formulated as follows.

$$
\begin{array}{lc}
\text { Objectives: } & \text { Maximizing } P, \\
& \text { Minimizing } \varepsilon_{1, \max } \\
& c_{i j}(i, j=1,2,3), \\
\text { Design } & \theta_{k}(k=1,2, \ldots \mathrm{nl}), \\
\text { variables: } & n c l \\
& \\
& \\
& -2.0 \leq c_{i j} \leq 2.0\left(\Delta c_{i j}=0.1\right), \\
& -75^{\circ} \leq \theta_{k} \leq 90\left(\Delta \theta_{k}=15^{\circ}\right), \\
& 0 \leq n c l \leq 16(\Delta n c l=2), \\
& P \geq 0, \\
& \varepsilon_{1, \max } \leq 11000 \mu(\text { around hole }), \\
& \varepsilon_{1, \max } \leq 5000 \mu(\text { other area) }
\end{array}
$$

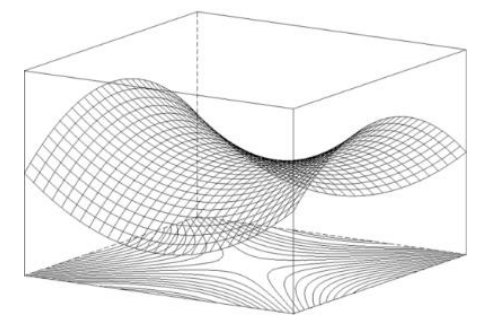

(a) Surface

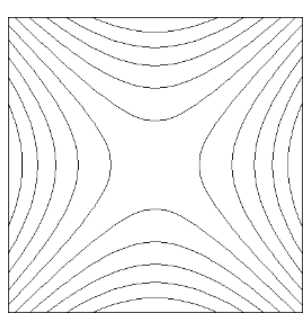

(b) Curvilinear shapes
Figure 5. (a) An example of a curved surface, and (b) the curvilinear fiber shape corresponding with curved surface of (a)

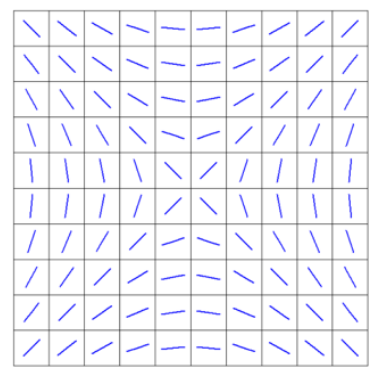

(a) $+\theta$ layer

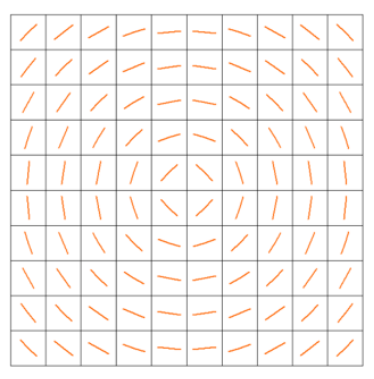

(b) $-\theta$ layer
Figure 6. The discrete curvilinear fiber shapes of $+\theta$ and $-\theta$ layers in FEA corresponding with Fig. 5

The objectives are minimizing the maximum principal strain $\varepsilon_{1, \max }$ and maximizing the productivity index $P$ which is defined as a function of curvature as follows.

$$
P=-3.5 \times 10^{5} \kappa^{2}+200 \kappa+30
$$

This relation is apporoximated by the experimental experiences and when the layer is composed of straight fibers, $P=31$.

The design variables are coefficients of cubic polynomial function $c_{i j}(i, j=1,2,3)$, fiber orientation angle $\theta_{k}$ in the $k$-th layer which has straight fibers only, and number of layer with curvilinear reinforcing fibers ncl.

Two elements adjacent to the hole are regarded as around hole elements and a less-restricted constraint is imposed compared with other parts. As an optimizer, the improved non-dominated sorted genetic algorithm (NSGA-II) [8] are used due to its high searching ability and versatility. 


\subsection{Optimization results}

Obtained optimum solutions are shown in Fig. 7. The vertical and horizontal lines indicate the productivity $P$ and the maximum principal strain $\varepsilon_{1, \max }$. The number of layers with curved fibers is distinguished by colors. It is known from Fig. 7 that the present study found widely distributed Pareto optimum solutions.

For example, the lay-up configuration of the solution $\mathrm{A}$ is $\left[+\theta /-\theta / 0 \%+\theta /-30 \%-\theta / 15^{\circ} /+\theta\right] \mathrm{s}$ where the $+\theta$ layer has the optimized coefficients of cubic polynomial $\left(c_{10}, c_{01}\right.$, $\left.c_{20}, c_{11}, c_{02}, c_{30}, c_{21}, c_{12}, c_{03}\right)=(-1.0,0.1,0.6,1.0,-0.4,-0.4$, $-1.3,-2.0)$, and its fiber shapes are given in Fig. 8 (a). The $-\theta$ layer has symmetric fiber shape with respect to the horizontal line. Distribution of principal strain $\varepsilon_{1}$ is demonstrated in Fig. 8 (b). The maximum value $\varepsilon_{1, \max }=$ $3976 \mu$ that is the minimum value in all obtained nondominated solutions, but the solution A has the lowest productivity $P=29.75$. On the other hand, the solution $B$ whose lay-up is $\left[-15^{\circ} \%+\theta / 0^{\circ} /-\theta /-15^{\circ} / 0^{\circ} / 30^{\circ} /-45^{\circ}\right]_{s}$ and coefficients are $c_{i j}=(-2.0,0.5,0.2,-1.7,-0.5,-1.9,-1.5$, 1.0) gives the higher principal strain $\varepsilon_{1, \max }=4225 \mu$ and productivity $P=30.75$ than the solution A. The solution C is only solution which is composed of straight fibers for all layers and its lay-up is $[-15 \% \% \% 15 \% \%$ $\left.45^{\circ}\right] \mathrm{s}$ with $\varepsilon_{1, \max }=4232 \mu$. This is the highest value in the obtained solutions even the straight part has mechanical properties superior to curvilinear fiber path. Therefore, the effectiveness of the curvilinear fibers with variable stiffnesses depending on curvature is confirmed from above.

\section{Conclusions}

The present study first experimentally revealed the relationship between curvature of reinforcing fiber and fiber volume fraction of laminated composites fabricated by the tailored fiber placement machine. It was found that the stiffness of the composite decrease as the curvature increases. Then, the obtained results are applied to the multi-objective optimization problem. From numerical results, it became clear that the composites with curved fibers have still advantages over those with straight fibers even their weakened stiffnesses due to fiber curvatures.

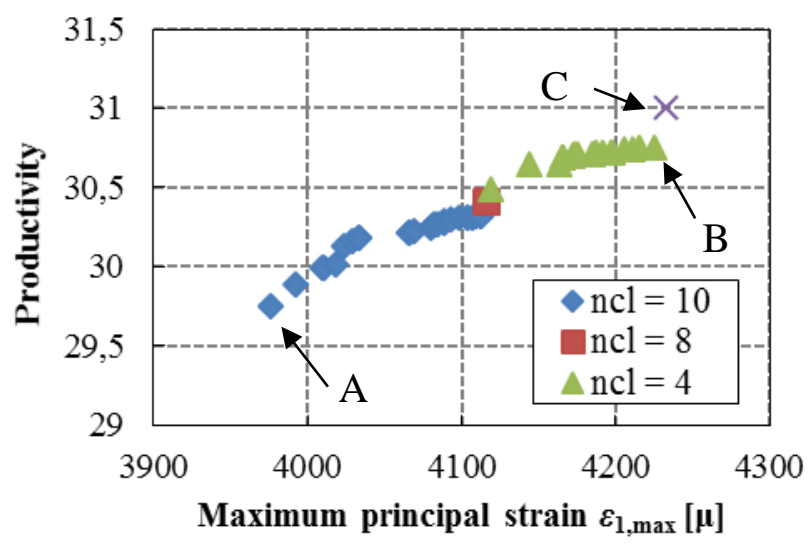

Figure 7. Obtained Pareto optimum solutions

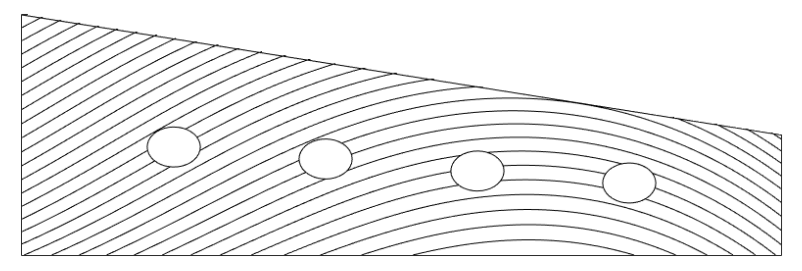

(a) Fiber shape for + layer, $P=29.75$

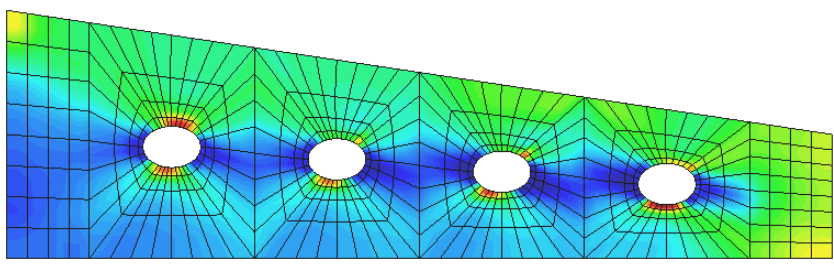

(b) Principal strain $\varepsilon_{1}$ distribution, $\varepsilon_{1, \max }=3976 \mu$

Figure 8. Fiber shape of + layer and strain distribution for solution A

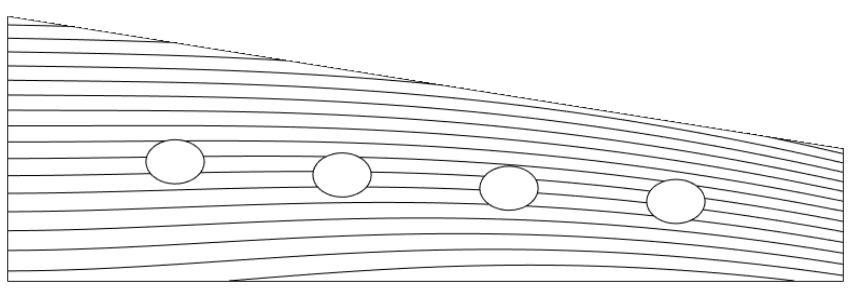

(a) Fiber shape for + layer, $P=30.75$

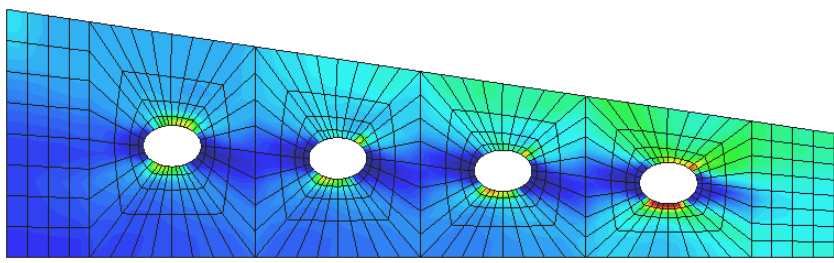

(b) Principal strain $\varepsilon_{1}$ distribution, $\varepsilon_{1, \text { max }}=4225 \mu$

Figure 9. Fiber shape of + layer and strain distribution for solution B

\section{References}

[1] Hyer, M. H. and Lee, H. H., The Use of Curvilinear Fiber Format to Improve Buckling Resistance of Composite Plates with Central Circular Holes, Composite Structures, Vol. 18(1991), pp. 239-261.

[2] Huang, J. and Haftka, R. T., Optimization of Fiber Orientation near a Hole for Increased Load-carrying Capacity of Composite Laminates, Structural Multidisciplinary Optimization, Vol. 30(2005), pp. 335-341.

[3] Parnas, L., Oral, S., and Ceyhan, Ü., Optimum Design of Composite Structures with Curved Fiber Courses, Composites Science and Structures, Vol. 63 (2003), pp. 8493-8516.

[4] Lopes, C. S., Gürdal, Z., and Camanho, P. P., Variable-stiffness Composite Panels: Buckling and First-ply Failure Improvements over Straight-fibre Laminates, Computer \& Structures, Vol. 86 (2008), pp. 897-907.

[5] Lopes, C. S., Camanho, P. P., Gürdal, Z., and Tatting B. F., Progressive Failure Analysis of Tow-placed, Variable-stiffness Composite Panels, International Journal of Solids and Structures, Vol. 44(2007), pp. 8493-8516.

[6] Honda, S., and Narita, Y., Vibration Design of Laminated Fibrous Composite Plates with Local Anisotropy Induced by Short Fibers and Curvilinear Fibers, Composite Structures, Vol. 93 (2011), pp. 902-910.

[7] Honda, S., Igarashi, T., and Narita, Y., Multi-Objective Optimization of Curvilinear Fiber Shapes for Laminated Composite 
Plates by Using NSGA-II", Composites Part B: Engineering, Vol. 45, 2013, 1071-1078.
[8] Deb, K., Multi-objective Optimization Using Evolutionary Algorithms, John Wiley and Sons Ltd, (2001). 